\title{
ACIDENTES DE TRABALHO NA EQUIPE DE ENFERMAGEM DE UM HOSPITAL DE ENSINO DO PARANÁ - BRASIL
}

WORK ACCIDENTS SUFFERED BY NURSING OF A STUDY HOSPITAL

IN PARANA - BRAZIL

ACCIDENTES DE TRABAJO EN EL EQUIPO DE ENFERMERIA DE UN HOSPITAL DE ENSEÑ ANZA DE PARANÁ - BRASIL

\section{IARA APARECIDA DE OLIVEIRA SÊCCO* MARIA LUCIA DO CARMO CRUZ ROBAZZI**}

\begin{abstract}
RESUMO
Trata-se de estudo epidemiológico descritivo, de delineamento transversal, cujo objetivo foi analisar os acidentes de trabalho típicos (ATTs) registrados pelos trabalhadores de enfermagem segundo as variáveis relacionadas ao tempo, ao espaço e à pessoa e estimar indicadores de risco. Teve como campo um hospital de ensino da região norte do Paraná (HE) e a população constituiu-se do número médio anual de 634,5 profissionais de enfermagem. A coleta de dados deu-se por meio das 392 notificações de acidentes de trabalho do período de 1997 a 2002, entre Comunicações de Acidentes de Trabalho e N otificações de Acidentes de Trabal ho com M aterial Biológico. Para a análise e discussão dos resultados foi preparado banco de dados com a utilização do Programa Epi-Info versão 6.04 C. Como resultados verificou-se que, dos 392 acidentes notificados, 89\% (349) foram típicos (ATTs) e apresentaram o Coeficiente de Risco M édio Anual (CRM A) igual a 9,2 acidentes para cada 100 trabalhadores. Os mais acometidos foram os Técnicos/Auxiliares de Enfermagem, com 93,9\% (328) dos casos eCRM A de 10,4. Os acidentes envolvendo exposição a materiais biológicos foram os mais presentes, com 64,2\% (224) das ocorrências, conferindo CRM A de 5,9; atingiram, principalmente, as mãos dos trabalhadores no manuseio de perfurocortantes. Constatou- se a necessidade de implementação de ações preventivas, com a revisão dos processos de trabalho, implementação das atividades de educação permanente e especial atenção na preven ção de doenças ocupacionais graves como Síndrome da I munodeficiência Adquirida eH epatite B e C.
\end{abstract}

Palabras chaves: Acidentes de trabalho, risco ocupacional, enfermagem.

\begin{abstract}
The present work is a descriptive epidemiologic study of transverse delineation. Its aim was to analyze typical work accidents (TWAs) reported by nursing employees according to variants in time, space and people, as well as to estimate risk indicators. It was carried out at a study hospital (SH) in the north region of Paraná and the population was made up of an annual average of 634.5 nursing professionals. Data collection was carried out through examination of 392 work accident reports since 1997 until 2002, which included Communication of Work Related Accidents and Notice of Work Related Accidents involving Biological M aterial. For analysis and discussion of the results, a database was prepared using Epi-info $6.04 \mathrm{C}$. The results showed that from 392 reported accidents, 89\% (349) were typical (TWAs) and presented Annual Average Risk Coefficient (AARC) of 9.2 accidents per 100 employees. The most affected were N ursing Technicians/Assistants, with $93.9 \%$ (328) of the cases and AARC of 10.4. Accidents involving exposure to biological materials were the most frequent $64.2 \%$ (224) of the reported ones- reaching an AARC of 5.9; they mainly affected the hands of the employees

* D ocente do Curso de Enfermagem na Universidade N orte do Paraná. Mestre em Saúde Coletiva. Doutoranda da Escola de Enfermagem de Ribeirão Preto - USP, Assessora Técnica da Diretoria de Enfermagem do Hospital Universitário Regional do Norte do Paraná (HURNP/UEL). Brasil. E-mail: <iarasecco@sercomtel.com.br>

** DocenteTitular da Escola deEnfermagem de Ribeirão Preto - Universidade de São Paulo, D outora em Enfermagem. E-mail: avrmlccr@glete.eerp.usp.br
\end{abstract}


during handling of needles and other sharp objects. It was observed the need for implementation of preventive actions such as revision of work processes, implementation of permanent educational activities and special attention in the prevention of serious occupational diseases such as Acquired Immunodeficiency Syndrome and Hepatitis B and C.

Keywords: Work accidents, occupational risk, nursing.

\section{RESUMEN}

Estudio epidemiológico descriptivo, de corte transversal, cuyo objetivo fue analizar los accidentes de trabajo típicos (ATTs) registrados por los trabajadores de enfermería según variables relacionadas al: tiempo, espacio, la persona y a estimar indicadores de riesgo. Fue realizado en un hospital de enseñanza de la región norte de Paraná (HE) y la población se constituyó del universo anual de 634,5 profesionales de enfermería. La recolección de datos se efectuó por medio de las 392 notificaciones de accidentes del trabajo del periodo de 1997 hasta 2002, entre Comunicaciones de Accidentes de Trabajo y Notificaciones de Accidentes de Trabajo con M aterial Biológico. Para el análisis y discusión de los resultados se elaboró una planilla de datos para la utilización del programa Epi-Info versión 6.04 C. Como resultados se verificó que, de los 392 accidentes notificados, un 89\% (349) fueron típicos (ATTs) y presentaron el Coeficiente de Riesgo M ediano Anual (CRM A) igual a 9,2 accidentes para cada 100 trabajadores. Los más afectados han sido los Técnicos/Auxiliares de Enfermería, con un 93,9\% (328) de los casos y CRM A de 10,4. Los accidentes producidos por la exposición a materiales biológicos fueron los más frecuentes, con un 64,2\% (224) de las ocurrencias, entregando CRM A de 5,9; afectaron, principalmente, las manos de los trabajadores en el manejo de material cortopunzante. Se constató la necesidad de implementación deacciones preventivas, en los procesos detrabajo, con actividades de educación permanente y especial atención en la prevención de enfermedades ocupacionales graves como Síndrome de la Inmunodeficiencia Adquirida y Hepatitis B y $C$.

Palabras claves: Accidentes de trabajo, riesgo ocupacional, enfermería.

Fecha recepción: 16/03/06. Fecha aceptación: 30/10/07.

\section{INTRODUÇÃO}

Hospitais são estruturas organizacionais complexas, decaráter médico esocial, destinadas a prestar serviços desaúdeà população, tanto na área curativa quanto preventiva, constituindosetambém em importantecentro educacional e de pesquisa em saúde. Eles estão integrados ao setor terciário da economia no Brasil e o seu adequado gerenciamento é fundamental para o seu bom desempenho, tanto no que diz respeito à qual idade dos serviços prestados aos usuários, quanto para o provimento de ade quadas condições de trabalho para os trabaIhadores que ali atuam. É fato que "saúde não tem preço, mas tem custo".

$A$ atenção à saúde no âmbito hospitalar é um trabalho coletivo, queenvolvea atuação de profissionais com saberes e práticas específicas das ciências da área da saúde e outras áreas administrativas e de apoio técnico e logístico. Os trabal hadores de enfermagem, que no Brasil somam aproximadamente $50 \%$ do contingente de profissionais atuantes no setor saúde (M inistério da Saúde Brasil, 2003), participam desse processo por meio de atividades relacionadasao cuidado em saúde, intervindo no processo saúde-doença tanto da coletividade, na dimensão do modelo epidemiológico, quanto do indivíduo, na dimensão do modelo médico-assistencial.

Os processos de trabal ho da enfermagem hospitalar, a forma como o trabalho de "cuidar da saúde dos outros" é dividido e organizado, conferem danos à integridade física e mental daqueles que provêem esses cuidados, causando-Ihes desgastes, devariadas naturezas eque, muitas vezes, trazem transtornos graves com repercussões pessoais e sociais de expressiva monta. Dentre os principais problemas 
decorrentes da atividade laboral desses profissionais estão os acidentes de trabal ho (ATs), e o conhecimento dosfatores de risco para essas ocorrências é importante ação de vigilância epidemiológica com vistas ao planejamento e efetivação de medidas preventivas (Silva A., 1996; Valenzuela, Sanhueza, RiquelmeeNuñez, 2002; Sêcco, 2002; M arziale, 2003).

Deacordo com a legislação brasileira, AT é aquele que ocorre no exercício do trabalho e quetraz como conseqüência uma lesão corporal ou perturbação funcional, com perda ou redução da capacidadepara o trabal ho, deforma permanente ou temporária, ou atémesmo a morte. É considerado como acidente de trabalho típico (ATT) aquele que ocorre durante o desempenho laboral, como acidentedetrajeto o que se dá durante o deslocamento entrea residência e o local de trabalho, como doença profissional aquela que foi produzida ou desencadeada pelo exercício do trabalho inerenteà atividade, e, como doença do trabalho a adquirida ou desencadeada por condições especiaisem queo trabalho érealizado equecom ele se relacione. Todo AT deve ser registrado na instância previdenciária competente, utilizando a Comunicação deAcidentes de TrabaIho (CAT) para este fim (Lei n. 8213, Brasil, 1991).

Os riscos ocupacionais a que os trabal hadores de enfermagem estão expostos no contexto do processo de trabalho hospitalar são denominados deriscosfísicos, biológicos, químicos, mecânicos, fisiológicos epsíquicos (Silva, 1996a).

Osriscos biológicostêm especial importância para os trabal hadores de enfermagem em razão do contato com sangue e outros fluidos corporais potencialmente infectantes, que podem incorrer em doenças graves como a Hepatite B, Hepatite $C$ e a Síndrome da Imunodeficiência Adquirida (AIDS). 0 risco de infecção pós-exposição ocupacional a material biológico, para exposição percutânea, éde0,3\% para o vírus da Imunodeficiência Adquirida (HIV), de 6 a $30 \%$ para o vírus da Hepatite $B$ (HBV) e de $1,8 \%$ para o vírus da Hepatite $C$ (HCV) (M elo et al., 2004). Os riscos mecânicos estão presentes no manuseio e transporte de pacientes obesos, na contenção de pacientes agressivos, no manejo e transporte de materiais e equipamentos volumosos e pesados. Os riscos fisiológicos expressam-se no trabaIho diuturno, nas longas jornadas em pé, pouco tempo para ir ao banheiro, no duplo emprego. Os riscosfísicos estão presentes no contato com as radiações, nos ruídos excessivos, nos problemas com a iluminação e altas temperaturas. Osriscosquímicos são encontrados no contato com desinfetantes, produtosfarmacêuticos diversos; quimioterápicos. Por fim, os riscos psíquicos são provenientes do contato com a dor ecom o sofrimento, no clima organizacional desfavorável, nos baixos salários, no desprestígio profissional, na resistência necessária a se manter no emprego (Bulhões, 1994; Sêcco, 2002; Silva, 1996).

A despeito de todas as dificuldades, é fato que os hospitais devem empreender esforços no sentido de melhorar as condições detrabaIho dos profissionais e, conseqüentemente, contribuir para a melhora na assistência à saúde prestada aos seus clientes. No Brasil, entre as normatizações que se aplicam diretamente aesteobjetivo, tem especial importânciaanova Norma Regulamentadora $\mathrm{N}$ o 32, que aborda as necessáriastransformações das condições de trabalho no setor saúde (Robazzi e M arziale, 2004; Robazzi e Barros Júnior, 2005).

0 estudo dosATs que acometem os trabaIhadores de enfermagem e, de maneira particular osATTs, apresenta-secomo importante instrumento de vigilância epidemiológica, devendo respaldar o planejamento e gerenciamento dos serviços de saúde no provimento de condições dignas de trabal ho para aqueles que constroem essa realidade. Este interesse justificou a realização da presente investigação. 


\section{OBJETIVO DO ESTUDO}

- Analisar o perfil epidemiológico dos ATTs ocorridos entre os trabalhadores de enfermagem registrados no hospital de ensino segundo: tipo, função exercida pelo acidentado, natureza do acidente, parte do corpo atingida, natureza da lesão, atividade desempenhada no momento do infortúnio, hora e número de horas trabalhadas até 0 momento da ocorrência;

- Estimar osindicadores derisco para oseventos.

\section{MATERIAL E METODOS}

Trata-se de um estudo descritivo, de delineamento do tipo transversal não controlado, sob o referencial teórico da epidemiologia clássica. Teve como campo um hospital de ensino da região norte do Paraná (HE), que caracteriza-se como hospital geral, terciário, público, com o total de 289 leitos. A população constituiu-sedetrabalhadores deenfermagem, com número médio anual de 634,5 profissionais, nas categorias de enfermeiro, técnico, auxiliar e atendente de enfermagem. Os dados foram coletados das 392 notificações de ATs registrados por meio das CATs e das N otificações de Acidentes de Trabalho com $M$ aterial Biológico (NATMBs), no período de 1997 a 2002. Quanto às NATM Bs, estas foram utilizadas de 1998 a 2002, ou seja, desde a implantação do Programa de Acidentes de Trabalho com M aterial Biológico (PATM B) na Instituição.

Os Coeficientes de Risco (CRs) apresentados referiram-se à razão percentual entre 0 número de acidentes ocorridos no mesmo local e período de tempo e o total de pessoas expostas às ocorrências (Laurenti, M ello H orge, Lebrão e Gotlieb, 1998). Designou-se como Coeficiente de Risco M édio Anual (CRM A) a média dos CRs dos seis anos do estudo e Razão de Risco M édia Anual a média da Razão de Risco do mesmo período
(CRM A1/CRM A2 = RRM A) para asvariáveis possíveis de quantificação. Para a análise e discussão dos resultados foi preparado um banco de dados com a utilização do Programa Epi-Info versão $6.04 \mathrm{C}$ e da Planilha M icrosoft Excel 2000, série Windows (M icrosoft, 1999).

O estudo foi aprovado pelo ComitêdeÉtica em Pesquisa do HE, conforme preconizado pela Resolução № 196/96 do Conselho Nacional de Saúde (M inistério de Saúde, 1996).

\section{RESULTAD OS}

As 392 notificações de ATs sofridos pelos trabalhadores de enfermagem entre os anos de 1997 a 2002 representaram 54,7\% dos 717 acidentes notificados em todo o hospital no referido período. Enquanto o CRM A do grupo dos trabal hadores de enfermagem foi estimado em 10,3 acidentes para cada 100 trabaIhadores, o CRM A dos demais grupos foi estimado em 5,0 para cada 100 trabalhadores. A RRM A entre os trabal hadores de enfermagem apresentou o valor de $2,1(10,3 / 5,0)$, ou seja, o risco desse pessoal acidentar, embora represente apenas $37,1 \%$ do contingente do $\mathrm{HE}$, éo dobro do risco de todosos outros trabalhadores, confirmando a relevância do problema.

OsATTs apresentaram a maior freqüência dos ATs e os mai ores CRs, totalizando $89,0 \%$ (349) das notificações e CRM A de 9,2, mostrando queda expressiva no último biênio. Quanto ao diagnóstico de doença profissional foi registrado em $1(\mu \mathrm{m})$ caso detuberculose pulmonar em 2001 e 16 casos no ano de 2002 , sendo estes referentes a surto de escabiosenorueguesa queacometeu os profissionais deenfermagem, o quetotalizou 4,3\% (17) dos registros para o período estudado eaumento crescentedos CRs. A Figura 1 apresenta a evolução anual dos CRs, de acordo com a classificação dos ATs. 


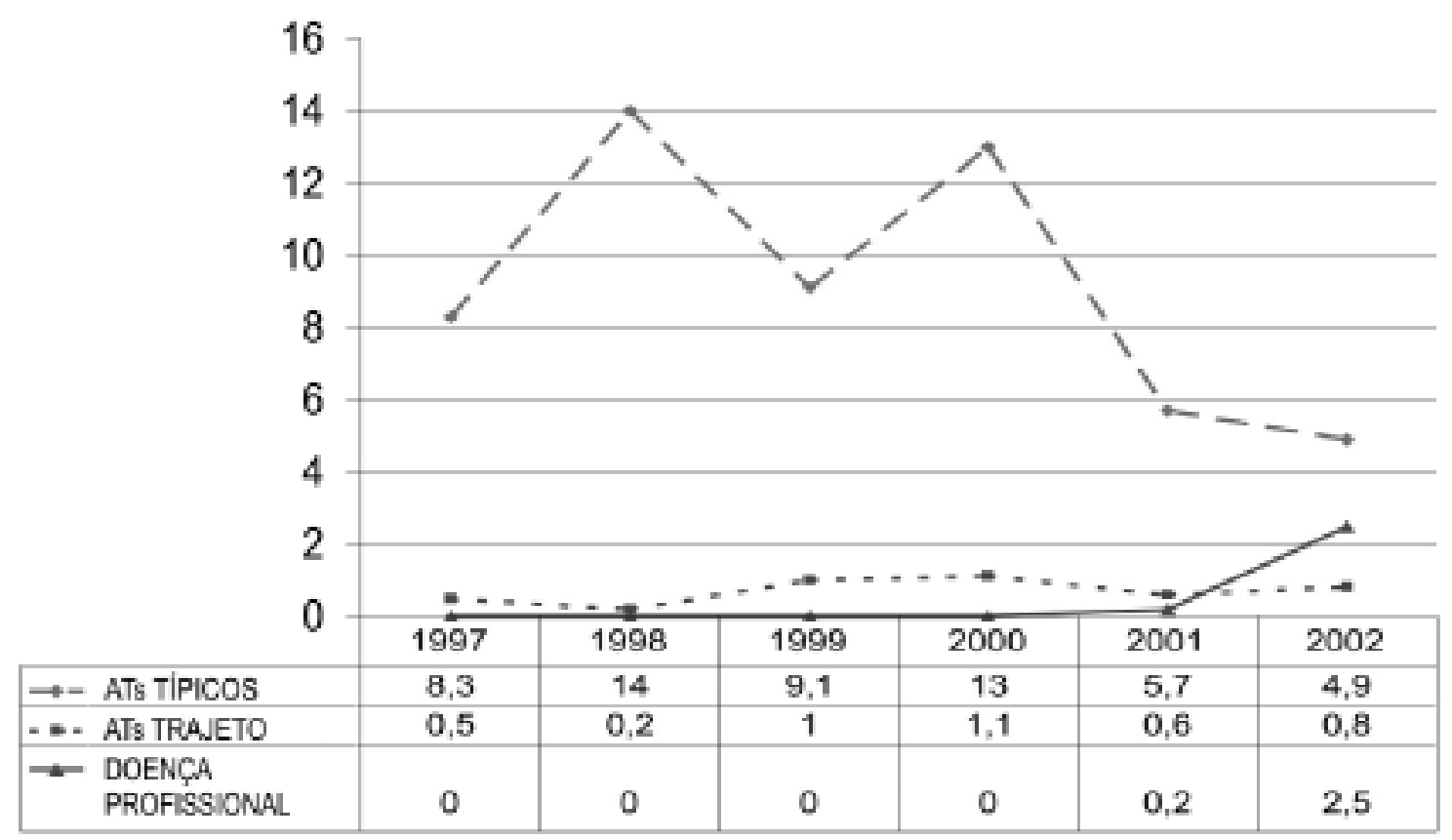

Figura 1. Distribuição do CR anual das notificações de ATTs da equipe deenfermagem do HE, segundo o tipo, de 1997 a 2002. Londrina, 2005.

A Tabela 1 apresenta as características dos ATTsocorridos entre os trabalhadores deenfermagem nos anos do estudo, ano a ano, conforme a proposta do estudo. Verificou-se que os técnicos/auxiliares foram os mais acometidos em todos os anos do estudo, respondendo por 93,9\% (328) dos casos e CRM A de 10,4 . Quanto à razão RRM A destes em relação aos enfermeiros, o valor estimado foi de $3,5(10,4 / 3,0)$, o que equivale dizer que o risco foi aproximadamente quatro vezes maior para a ocorrência de ATTs para os técnicos/ auxiliares quando comparado com os profis- sionais de enfermagem. $\mathrm{Na}$ classificação dos ATTs, com relação à natureza do acidente, verificou-se que os casos deATM Bs estiveram presentes em $64,2 \%$ (224) das ocorrências, conferindo CRMA de 5,9. Os ATM Bs mostraram-se de grande relevância para a equipe em relação a todos os demais ATTs, sendo confirmado pelos resultados da RRM A que foi estimada em $1,8(5,9 / 3,3)$. Portanto, o risco da ocorrência de um ATM B foi em torno de $80 \%$ maior do que a ocorrência de qualquer outro tipo de acidente no desenvolvimento da atividade laboral de enfermagem. 
Tabela 1. Notificações de ATTs entre trabalhadores de enfermagem, segundo a função, natureza do acidente, parte do corpo atingida e natureza da lesão, de 1997 a 2002. Londrina, 2005.

\begin{tabular}{|c|c|c|c|c|c|c|c|c|c|c|c|}
\hline \multirow[b]{2}{*}{ FUNÇÃO } & \multirow{2}{*}{$\begin{array}{c}1997 \\
\mathrm{n}\end{array}$} & \multicolumn{2}{|c|}{19981999} & \multirow{2}{*}{$\begin{array}{c}2000 \\
n\end{array}$} & \multirow{2}{*}{$\begin{array}{c}2001 \\
n\end{array}$} & \multirow{2}{*}{$\begin{array}{c}2002 \\
\mathrm{n}\end{array}$} & \multicolumn{2}{|c|}{ TOTAL } & \multicolumn{3}{|c|}{ MÉDIA ANUAL } \\
\hline & & $\mathbf{n}$ & $\mathbf{n}$ & & & & $\mathbf{n}$ & $\%$ & $\mathbf{n}$ & № trab.* & CR \\
\hline Técnico/Auxiliar enf. & 52 & 85 & 51 & 76 & 32 & 32 & 328 & 93,9 & 54,7 & 524,2 & 10,4 \\
\hline Atendente enf. & 1 & 1 & 1 & 1 & 0 & 0 & 4 & 1,2 & 0,7 & 14,3 & 4,7 \\
\hline Enfermeiro & 0 & 6 & 3 & 4 & 4 & 0 & 17 & 4,9 & 2,8 & 96,0 & 3,0 \\
\hline TOTAL & 53 & 92 & 55 & 81 & 36 & 32 & 349 & 100 & 58,2 & 634,5 & 9,2 \\
\hline NATUREZA DO ACIDENTE & $\mathbf{n}$ & $\mathbf{n}$ & $\mathbf{n}$ & $\mathbf{n}$ & $\mathbf{n}$ & $\mathbf{n}$ & $\mathbf{n}$ & $\%$ & $\mathbf{n}$ & № Trab. & CR \\
\hline Contato com material biológico & 22 & 62 & 42 & 53 & 27 & 18 & 224 & 64,2 & 37,3 & 634,5 & 5,9 \\
\hline Impactos & 12 & 11 & 2 & 13 & 2 & 6 & 46 & 13,1 & 7,7 & 634,5 & 1,2 \\
\hline Contato material médico-hospitalar & 14 & 17 & 6 & 6 & 1 & 1 & 45 & 12,9 & 7,5 & 634,5 & 1,2 \\
\hline Esforço excessivo/Inadequado & 2 & 2 & 2 & 3 & 4 & 5 & 18 & 5,2 & 3,0 & 634,5 & 0,5 \\
\hline Quedas & 1 & 0 & 1 & 4 & 2 & 2 & 10 & 2,9 & 1,7 & 634,5 & 0,3 \\
\hline Outros & 2 & 0 & 2 & 2 & 0 & 0 & 6 & 1,7 & 1,0 & 634,5 & 0,2 \\
\hline TOTAL & 53 & 92 & 55 & 81 & 36 & 32 & 349 & 100 & 58,2 & 634,5 & 9,2 \\
\hline PARTE DO CORPO ATINGIDA & $\mathbf{n}$ & $\mathbf{n}$ & $\mathbf{n}$ & $\mathbf{n}$ & $\mathbf{n}$ & $\mathbf{n}$ & $\mathbf{n}$ & $\%$ & $\mathbf{n}$ & № Trab. & CR \\
\hline Mãos & 40 & 70 & 38 & 57 & 22 & 19 & 246 & 70,5 & 41,0 & 634,5 & 6,5 \\
\hline Olhos & 3 & 12 & 7 & 7 & 8 & 4 & 41 & 11,7 & 6,8 & 634,5 & 1,1 \\
\hline Membros inferiores & 6 & 2 & 1 & 3 & 2 & 1 & 15 & 4,3 & 2,5 & 634,5 & 0,4 \\
\hline Tronco & 2 & 2 & 2 & 1 & 4 & 6 & 17 & 4,9 & 2,8 & 634,5 & 0,4 \\
\hline Outras partes & 2 & 6 & 7 & 13 & 0 & 2 & 30 & 8,6 & 5,9 & 634,5 & 0,8 \\
\hline TOTAL & 53 & 92 & 55 & 81 & 36 & 32 & 349 & 100 & 58,2 & 634,5 & 9,2 \\
\hline NATUREZA DA LESÃO & $\mathbf{n}$ & $\mathbf{n}$ & $\mathbf{n}$ & $\mathbf{n}$ & $\mathbf{n}$ & $\mathbf{n}$ & $\mathbf{n}$ & $\%$ & $\mathbf{n}$ & № Trab. & CR \\
\hline Perfuração & 23 & 57 & 33 & 47 & 18 & 11 & 189 & 54,2 & 31,5 & 634,5 & 5,0 \\
\hline Corte & 13 & 13 & 3 & 8 & 3 & 5 & 45 & 12,9 & 7,5 & 634,5 & 1,2 \\
\hline Contusão & 6 & 3 & 3 & 9 & 4 & 3 & 28 & 8,0 & 4,7 & 634,5 & 0,7 \\
\hline Irritação nos olhos & 1 & 3 & 2 & 1 & 8 & 5 & 20 & 5,8 & 3,3 & 634,5 & 0,5 \\
\hline Entorse ou torção & 0 & 0 & 0 & 1 & 2 & 4 & 7 & 2,0 & 1,2 & 634,5 & 0,2 \\
\hline Escoriação & 2 & 1 & 1 & 0 & 0 & 0 & 4 & 1,1 & 0,7 & 634,5 & 0,1 \\
\hline Distensão muscular & 0 & 1 & 0 & 2 & 1 & 0 & 4 & 1,1 & 0,7 & 634,5 & 0,1 \\
\hline Luxação & 2 & 0 & 0 & 2 & 0 & 0 & 4 & 1,1 & 0,7 & 634,5 & 0,1 \\
\hline Outros & 6 & 14 & 13 & 11 & 0 & 4 & 48 & 13,8 & 8,0 & 634,5 & 1,2 \\
\hline TOTAL & 53 & 92 & 55 & 81 & 36 & 32 & 349 & 100 & 58,2 & 634,5 & 9,2 \\
\hline
\end{tabular}

* Distribuição dos 634,5 trabal hadores de enfermagem por função, de 1997, 1998, 1999, 2000, 2001 e 2002, respectivamente: enfermeiros (94, 97, 96, 97, 96, 96), com média anual de 96 profissionais; técnicos/auxiliares de enfermagem $(530,526,492,532,526,539)$, com média anual de 524,2 e atendentes (17, 15, 15, 13, 13, 13), com média anual de 14,3. (Hospital, 2004). 
Os impactos deram-se em 13,2\% (46) dos eventos, conferindo CRMA de 1,2. Foram eventos ocorridos em manuseio de equipamentos, pelo impacto do corpo com o mobiliário em ambientes com espaço exíguo, entre outros. Dois dos casos foram ocasionados por agressão de paciente com agitação psicomotora ao trabalhador de enfermagem.

Em relação à parte do corpo atingida, verificou-se que as mãos dos trabalhadores estiveram mais expostas a ATTs em todos os anos do estudo, totalizando 70,5\% (246) dos casos e CRM A anual igual a 6,5, o que não difere da realidade dos ATTs em todas as demais áreas produtivas. A RRM A dos aciden- tes envolvendo as mãos dostrabalhadores com relação às demais partes foi de $2,4(6,5 / 2,7)$, ou seja, mais que o dobro, o que reforça a re levância do problema no contexto dos ATTs na enfermagem (Tabela 1; Figura 2).

Chama a atenção que em $11,7 \%$ (41) dos acidentes os olhos foram acometidos e em $85,4 \%$ (35) destes houve contato direto com materiais biológicos; em 13 casos $(37,1 \%)$ houve contato com sangue, sendo 2 por paciente soropositivo para HIV e outro para o HVB; em 3 casos (8,6\%) a mucosa ocular foi atingida por secreção pulmonar e em outros três $(8,6 \%)$ houve respingo de urina de paciente.

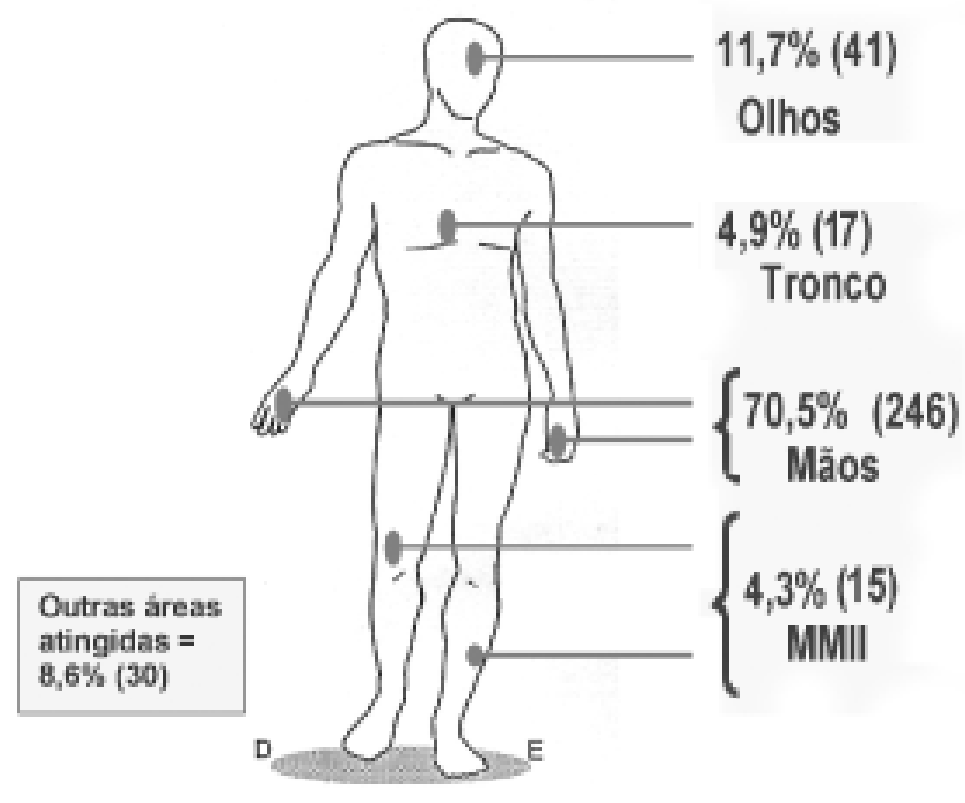

Figura 2. Notificações deATTs da equipe de enfermagem segundo a área do corpo atingida no acidente, de 1997 a 2002. Londrina, 2005.

As lesões com perfurações foram as mais freqüentes entre os trabalhadores deenfermagem do H ospital, estando ligadas di retamente ao manuseio de agul has tanto na assistência direta aos pacientes quanto nas tarefas de preparo de materiais e descarte das mesmas. Do total de 349 ATTs ocorridos, 169 (48,4\%) foram provocados por agulhas hipodérmicas, sendo que em 14 eventos (8,2\%) estas haviam sido utilizadas em portadores deHIV; em 
um caso em pacienteportador deH CV eHIV, simultaneamente, e em um caso em paciente portador de HCV.

O CRM A igual a 5,0 foi o encontrado para as lesões com perfurações nas mãos do trabaIhador. A RRM A estimada entre os casos de lesões por perfuração em relação às lesões por corte, segundo tipo de agravo mais freqüente notificado, apresentou o valor de $4,2(5,0 / 1,2)$, o que demonstra a relevância deste tipo de acidente para o pessoal de enfermagem.

Lesões osteomusculares ocorridas em razão dos ATTs deram-se em 28 casos de contusão, 1 caso de fratura, 7 de entorse, 4 de luxação, 4 de distensão muscular. Somaram $12,3 \%$ (43) dos casos de ATTs, cujo CR foi estimado em 1,1.

No que se refere à atividade desenvolvida no momento do acidente, os resultados de- monstraram que aquelas que apresentaram maior risco foram aquelas relacionadas aos procedimentos deenfermagem na assistência direta aos pacientes, como era esperado. A freqüência apresentada foi de 35,2\%, conferindo assim o CRM A de 3,2.

0 expressivo número de ocorrências de acidentes no manuseio demateriais contaminados nas atividades de processamento de instrumental e reutilizáveis, como os desenvolvidos nas salas de desinfecção demateriais dos setores e do Centro de M aterial, totalizaram 13,8\% (48) das ocorrências, conferindo CRM A de 1,3, tendo a mesma expressão que o CR das tarefas de punção venosa, já reconhecidas como atividades de alto risco para ATTs para o pessoal de enfermagem (Tabela 2).

Tabela 2. Notificações de ATTs entre trabalhadores de enfermagem, segundo a atividade desempenhada no momento do acidente, de 1997 a 2002. Londrina, 2005.

\begin{tabular}{|c|c|c|c|c|c|c|c|c|c|c|c|}
\hline \multirow{2}{*}{$\begin{array}{l}\text { Atividade desempenhada } \\
\text { no momento do acidente }\end{array}$} & \multirow{2}{*}{$\begin{array}{c}1997 \\
n\end{array}$} & \multirow{2}{*}{$\frac{1998}{n}$} & \multirow{2}{*}{$\frac{1999}{n}$} & \multirow{2}{*}{$\frac{2000}{n}$} & \multirow{2}{*}{$\begin{array}{c}2001 \\
n\end{array}$} & \multirow{2}{*}{$\frac{2002}{n}$} & \multicolumn{2}{|c|}{ TOTAL } & \multicolumn{3}{|c|}{ Média anual } \\
\hline & & & & & & & $\mathbf{n}$ & $\%$ & $n$ & $\%$ & CR \\
\hline Procedim. enfermagem & 15 & 18 & 30 & 33 & 9 & 18 & 123 & 35,2 & 20,5 & 634,5 & 3,2 \\
\hline Punção venosa & 6 & 16 & 5 & 11 & 7 & 4 & 49 & 14,0 & 8,2 & 634,5 & 1,3 \\
\hline $\begin{array}{l}\text { Manuseio de material } \\
\text { contaminado }\end{array}$ & 11 & 15 & 4 & 9 & 4 & 5 & 48 & 13,7 & 8,0 & 634,5 & 1,3 \\
\hline Admin. medicamentos & 6 & 13 & 6 & 9 & 3 & 2 & 39 & 11,2 & 6,5 & 634,5 & 1,0 \\
\hline Descarte agulha cont. & 3 & 6 & 3 & 4 & 0 & 1 & 17 & 4,9 & 2,8 & 634,5 & 0,4 \\
\hline $\begin{array}{l}\text { Punção p/ glicemia de } \\
\text { polpa digital }\end{array}$ & 0 & 6 & 3 & 4 & 3 & 1 & 17 & 4,9 & 2,8 & 634,5 & 0,4 \\
\hline Manuseio mat. limpo & 5 & 7 & 1 & 3 & 0 & 0 & 16 & 4,6 & 2,7 & 634,5 & 0,4 \\
\hline Instrumentação cir. & 0 & 6 & 1 & 2 & 1 & 0 & 10 & 2,9 & 1,7 & 634,5 & 0,3 \\
\hline Retirada venóclise & 1 & 0 & 0 & 0 & 5 & 0 & 6 & 1,7 & 1,0 & 634,5 & 0,2 \\
\hline Encape de agulha & 0 & 1 & 0 & 2 & 0 & 0 & 3 & 0,9 & 0,5 & 634,5 & 0,1 \\
\hline Sem referência & 6 & 4 & 2 & 4 & 4 & 1 & 21 & 6,0 & 3,5 & 634,5 & 0,6 \\
\hline TOTAL & 53 & 92 & 55 & 81 & 36 & 32 & 349 & 100 & 58,2 & 634,5 & 9,2 \\
\hline
\end{tabular}

Ostrabalhadores de enfermagem realizam jornada detrabalho de 36 horas semanais. Em torno de $60,5 \%$ (384) do pessoal atua em se- tores deturnosininterruptos, totalizando seis horas diárias (manhã, das 7 às 13 horas e tarde, das 13 às 19 horas) com um plantão de 12 
horas no sábado ou domingo ( 7 às 19 horas); $31,1 \%$ (198) no sistema de turnos noturnos de 12 horas com 36 horas de descanso (das 19 às 7 horas). Os demais 8,4\% (53) permaneceram lotados em setores do horário diurno de 8 horas, de segunda a sexta-feira, tais como os ambulatórios, setor de radiologia e quimioterapia (H ospital, 2004).

Os resultados relativos à hora conhecida da ocorrência do acidente mostraram que a maioria dos eventos deu-se entre $9,10,12,15$, 16, 18, 22 e 6 horas. Chama a atenção o fato de que os horários que antecedem a passagem de plantão apresentaram elevação no número de ocorrências (12, 18 e 6 horas). Observou-se que $72,3 \%$ (240) dos eventos atingiram os trabalhadores do período diurno. Em 27,7\% (92) dos acidentes os acometidos atuavam no turno da noitee, em apenas 7,4\% (26) dos casos, os acidentados atuavam em unidades com turnos de 8 horas.

A maioria dos casos de ATTs ocorreu entre a terceira e a quinta hora trabalhada do turno diurno eentrea tercei ra ea quarta hora do noturno. 0 turno da noite apresentou menores números de notificações nas horas mais avançadas, exceto às 2 horas da manhã. Contudo, entre 5 e 6 horas, ao término do turno, a situação se reverteu, aumentando as ocorrências.

\section{DISCUSSÃO}

H ospitais são tidos pela sociedade como provedores de saúde para a população, em diferentes níveis de complexidade. Contudo, são importante fonte de riscos para os seus trabalhadores. No contexto do trabalho hospitalar, a equipe de enfermagem está particularmente exposta e, conseqüentemente, mais vulnerável a infortúnios, uma vez que a característica da profissão é a assistência direta aos pacientes, o cuidado de enfermagem. Estimar os indicadores de risco é fundamental para 0 adequado acompanhamento desta si- tuação, numa tarefa de "vigília" e"ação" constantes.

Osresultados do presenteestudo corroboraram os apresentados por outrosautores, que confirmaram a relevância dos acidentes que acometem esses trabalhadores no contexto dos hospitais e, portanto, devem ser merecedores de atenção no que se refere à revisão dos processos de trabalho e implementação de medidas preventivas (Oliveira, M akaron e Morrone, 1982; M onteiro, Carnio, Alexandre, 1987; Benatti, 1997; Tomasina e Gómez Etchebarne, 2001; Brevidelli e Cianciarullo, 2002; Sêcco, Robazzi, Shimizu eRubio, 2005).

$\mathrm{Na}$ equipe de enfermagem, a expressiva maioria de ATTs em relação aos de trajeto e doenças profissionais ficou dentro do esperado, uma vez queestemesmo cenário seapresenta nas estatísticas oficiais do M inistério da Previdência Social no Brasil, assim como em outros estudos que investigaram estes eventos na enfermagem (Silva, 1998; Benatti, 1997; M arziale, 2003; Valenzuela, Sanhueza, Riquelme \& Nuñez, 2002; M inistério da Previdência Social, Brasil, 2005).

Quanto à diminuição do CR dos ATTs no último biênio do estudo, épossível inferir que este resultado esteja relacionado com a meIhora nos processos de trabalho e/ou com a prevenção dos acidentes, produto de trabaIho de capacitação realizado por unidades educativas da I nstituição, tais como a Comissão de Controle de Infecção Hospitalar, Comissão Interna de Prevenção de Acidentes, Seção de Educação e Pesquisa.

Contudo, é possível que seja uma reação à implantação do PATM B, em 1998. Este, num primeiro momento, podeter sido o elemento propulsor do maior número de notificações deATM B no período entre 1998 e 2000, contribuindo para o aumento dos CRs para os anos respectivos. Verificou-se, no seu desenrolar, queo Programa trouxedificuldades para o processo de registro dos eventos, com aumento da burocracia, problemas no fluxo de atendimento médico, laboratório, farmácia, até 0 atendimento no órgão do Sistema de 
Saúde Pública encarregado de dar continuidade ao tratamento. Estes problemas podem estar associados, ainda, a outros já conhecidos, relacionados à dificuldade de adesão à quimioprofilaxia pós-exposição ocupacional, tema estudado por diversos pesquisadores brasileiros (Almeida, 2003; Bál samo, 2002; Miola, 2005; Sailer, 2004).

Os resultados mostraram que os técnicos/ auxiliares de enfermagem foram os queapresentaram maiores CRs para ATTs em todos os anos do estudo, situação já esperada dada a maior proximidade desses trabalhadores com os pacientes, na assistência direta. A eles são atribuídas as tarefas de higienização econforto, administração de medicamentos, processamento demateriais, manuseio etransporte de equipamentos, desinfecção e preparo e esterilização de materiais cirúrgicos e instrumentais diversos, entre outras do "fazer em enfermagem" (Benatti, 1997; Brandi Benatti eAlexandre, 1998; Sarquis, 1999; Silva, 1998).

Estudos esclareceram que os enfermeiros, tal como na presente investigação, ficam preservados das ocorrências de ATTs por estarem mais distanciados do cuidado, em razão das tarefas gerenciais que a eles são atribuídas (Benatti, 1997; Sarquis, 1999; Silva V., 1996). Outra possibilidade, e para justificar estes resultados, éa proposição de que os enfermeiros, detentores de maior capacitação técnico-científica estariam menos sujeitos às ocorrências (M arziale, 2003; Silva, 1998). Contudo, verificou-seque os ATs que acometeram os enfermeiros deram-se nas unidades em que estes têm maior contato com os pacientes, realizam assistência direta, como as Unidades deEmergência edeTratamento Intensivo, sinalizando que o diferencial possa estar ligado ao tipo de tarefa desenvolvida e não necessariamente à função.

Destaca-sequeosatendentes, por impedimentos legais em razão da menor qualificação profissional, foram impedidos de prestar atendimento direto aos pacientes e de realizar tarefas de enfermagem de maior complexidade, o que, possivelmente, tenha contribu- ído para a diminuição dos índices de risco ocupacional entre eles (Decreto n. 94.406, Brasil, 1987).

Asmãos dos trabal hadores deenfermagem foram a parte do corpo queincorreu em maiores riscospara os ATTs, reafirmando o quanto a atividade tem caráter manual. Assim como em todos os estudos que se ocuparam desta avaliação, ficou demonstrado o quanto esse caráter éexpressivo na atividade de prestação de assistência nos serviços desaúde(Silva, 1998; Jansen, 1997; Brandi et al.,1998; Marziale, 2003; M arziale e Robazzi, 2004).

Os trabalhadores de enfermagem foram acometidos, de maneira hegemônica, por ATM B , o que reforça a necessidade de atenção especial a todos os processos de trabal ho que incorrem em riscos biológicos. A maioria das exposições teve como elemento causador as agulhas hipodérmicas. Tais situações, muitas vezes, são consideradas inerentes à profissão, sendo subestimadas pelos trabalhadores de enfermagem. São ocorrências relacionadas às atividades de punções venosas, administração de medicamentos, retirada de venóclises, descarte no lixo perfurocortante, desinfecção de instrumental cirúrgico, organização e limpeza de mesas cirúrgicas, entre outros.

Faz-se necessário considerar também o agravante de acidentes provocados por aguIhas deixadas inadvertidamente em meio a campos cirúrgicos, misturados ao material nos pacotes de curativos, equeterminam por ferir o trabal hador, os quais, mesmo utilizando equipamento de proteção individual, acabam se acidentando. Em que pese a realização de programas educacionais nas instituições, o fato é que o problema dos materiais perfurantes e cortantes persiste entre os trabalhadores de enfermagem, de maneira bastante expressiva.

Os dados revelam que, aparentemente, a prática de reencape de agulha tem se mostrado com valores diminuídos nos últimos anos com relação a outros estudos já realizados no $\mathrm{HE}$, o que leva a crer que as novas técnicas 
estão incorporadas à prática do pessoal de enfermagem.

O segundo tipo de agravo com maior indicador de risco foram os problemas osteomusculares como contusões, entorses ou torções que também se fizeram expressivos neste estudo, assim como em outras investigações (Benatti, 1997; Jansen, 1997; M arziale, 2003; Parada, 2002; Silva, 1998). São conseqüência da necessidade de mobilização e transporte de pacientes obesos, de quedas sofridas em pisos escorregadios, da local ização de materiais e equipamentos em armários altos, do espaço exíguo para mobiliários. Digno denota éque, ao contrário das perfurações, as doresna coluna advindas demovimentos bruscos, necessidade de segurar um paciente, com força, para que não caia, as quedas, os impactos sofridos, apresentam-se de maneira mais intensa no corpo do trabalhador, que acaba sendo impelido a registrar o fato pois, muitas vezes, 0 acidente 0 impossibilita de trabalhar.

Os resultados evidenciaram que os horários imediatamente anteriores à passagem de plantão (6, 12 e 18 horas) apresentam maiores riscos, possivelmente pela maior intensidade do trabalho visando o término das tare fas. Contudo, no momento da troca de turnos, da denominada passagem de plantão, às 7, 13 e 19 horas, observou-se menores freqüências de ATTs, o que, possivelmente, reflita a desaceleração do ritmo e volume de trabalho. Oshorários compreen didos nos turnos da manhã e da tarde mostram que o trabalho permanece intenso em todo o período subseqüente a estes horários, possivelmente em razão do movimento do PS, CC, procedimentos diversose transportede pacientes para exames. As primeiras horas da noite apresentam maior intensidade de trabalho também no PS enas unidades de internação por causa do retorno dos pacientes do CC, como também devido às tarefas de administração de medicamentos que se dão nesse horário visando diminuir seu volume durante o período noturno para evitar que o paciente seja incomodado durante o sono.
O quanto o trabalho noturno em hospitais é nocivo para a saúde do trabalhador de enfermagem étemática para muitos estudiosos. Em que pese a importância desse profissional para os pacientes, éfato que o trabalho noturno interfereno ritmo biológico dostrabalhadores, no seu estado de alerta cuja perdatorna-seimportante fator deATs (Bulhões, 1994).

Ponto importantea ser observado na análise do número de horas trabalhadas até a ocorrência do acidente é a sobrecarga de atividades desenvolvidas em outros serviços, ou atémesmo as atividades do lar, tão marcantes para as mulheres. 0 que se registra ser a segunda ou a terceira hora trabalhada em um hospital, na verdade podeser a 14a ou 15a hora deatividadedo trabal hador, marcada por uma previsível fadiga, por reflexos diminuídos, pelo sono, pela irritabilidade e outros fatores que, indubitavelmente, podem incorrer em riscos não só para os profissionais mas também para os pacientes atendidos.

Em síntese, observou-se que as realidades do trabal ho deenfermagem diferem muito de acordo com a maneira como é realizado e como estão constituídas as equipes, sendo necessário conhecêlas para identificar os riscos e, conseqüentemente, estabelecer cargas específicas de trabal ho, bem como desenvolver estratégias preventivas adequadas.

\section{CONSIDERAÇÕES FINAIS}

A presenteinvestigação dosATTspossibilitou estimar indicadores de risco para as ocorrências, ano a ano, considerando-seque estes são importantesinstrumentos devigilância epidemiológica para o Hospital e todas as instâncias interessadas na preservação e promoção da saúde do trabal hador de enfermagem.

Os achados evidenciaram que os ATTs apresentaram-se hegemônicos em todos os anos, sendo que os técnicos/auxiliares de enfermagem foram os que apresentaram maiores CRs, possivelmente em razão da proximi- 
dade conferida pela assistência direta aos pacientes.

Os ATM Bs foram os que apresentaram maiores indicadores de riscos, sendo que as mãos acometidas por perfurações foram as lesões de maior risco, tendo as agulhas papel de destaque como causa das mesmas. 0 período diurno e primeiras horas do noturno mostraram maior freqüência de ATTs, especialmente no PS, CC e Unidade de Terapia Intensiva Adulto.

Ressalta-sea importância demedidas preventivas por meio de estratégias educativas e revisão dos processos de trabalho para prevenir os acidentes. I gualmente importante é o acompanhamento da situação de saúde dos trabalhadores de enfermagem pelo Serviço de M edicina O cupacional, levando-se em conta as peculiaridades do envel hecimento desta população, assim como as características de suas condições de vida.

A saúde do trabalhador de enfermagem deve tratar-se de interesse dos trabal hadores, das entidades de classe, dos sindicatos, dos empresários e gestores dos serviços, das escolas de enfermagem, e, sobretudo, dos usuários dos serviços que, espera-se, sejam os primeirosa buscar atendimento por pessoas em condições dignas de trabalho e de vida, que tenham capacidade efetiva de realizar um trabalho de qualidade, pautado na responsabilidade, na competência técnico-científica e na ética.

\section{REFERÊNCIAS}

Almeida, C.A.F. (2003). Acidente do trabalho: adesão a quimioprofilaxia dos trabalhadores da saúde pós-exposição a material biológico humano. Dissertação de Mestrado, UniversidadeEstadual deCampinas, Campinas, Brasil.

Bálsamo, A.C. (2002). Estudo sobreos acidentes de trabalho com exposição aos líquidos corporais humanos em trabalhadores de saúde. Dissertação deM estrado, Escola deEnfermagem da Universidade deSão Paulo, São Paulo, Brasil.
Benatti, M .C.C. (1997). Acidente do trabal ho em um Hospital Universitário: um estudo sobrea ocorrência e os fatores de risco entre trabaIhadores de enfermagem. Tese de D outorado, Escola de Enfermagem de Ribeirão Preto da Universidade de São Paulo, Ribeirão Preto, Brasil.

Brandi, S.; Benatti, M.C. \& Alexandre, N.M.C. (1998). O corrências de acidentes de trabalho por material perfurocortante entre trabalhadores de enfermagem de um H ospital Universitário da cidade de Campinas, Estado de São Paulo. Rev. Esc. Enfermagem, 32(2), 124-133.

Brevidelli, M .M .\& Cianciarullo, T.I. (2002). Análise dos acidentes com agulhas em um hospital universitário: situações deocorrência etendências. Rev. Latino-Am. Enfermagem, 10 (6), 780-786.

Brasil (1987). Decreto № 94.406, de 8 de junho de 1987. (1987) Regulamentada a Lei ㅇ․ 7498 de 25 de junho de 1986. Diário Oficial da União, Brasília, 9/jun./1987. Dispõe sobre o Exercício da Enfermagem e dá outras providências.

Brasil (1991). Lei n. 8213 de 24 de julho de 1991. Diário Oficial daUnião, Brasília, 14/ago./1998. Dispõe sobre os Planos de Benefícios da Previdência Social e dá outras providências. Disponível em: <http://www81.dataprev.gov.br/ sislex/paginas/42/1991/8213.htm>.Acesso em 04/jul./2005.

Bulhões, I. (1994). Riscos do trabalho em enfermagem. Rio de Janeiro, Brasil. [s.n.].

Hospital Universitário Regional do Norte Do Paraná (2004). Divisão deRecursos Humanos. Relatório quantitativo dos servidores do Hospital Universitário Regional do Norte do Paraná. Londrina, Brasil. M aterial mimeografado.

Jansen, A.C. (1997). Um novo olhar para acidentes de trabalho na enfermagem: a questão do ensino. Dissertação de Mestrado, Escola de Enfermagem de Ribeirão Preto da Universidade de São Paulo, Ribeirão Preto, Brasil.

Laurenti, R.; M ello Horge, M.H.P.; Lebrão, M .L. \& Gotlieb, S.L.D. (1998). Estatísticas de Saúde. São Paulo Brasil. Ed. EPU/Edusp.

M arziale, M .H .P. (2003). Ocurrencia de accidentes de trabajo causados por material cortopunzante entre trabajadores de enfermería en 
hospitales de la región nordeste de São Paulo, Brasil. Ciência y Enfermeria 9(1), 21-30.

Marziale, M.H.P \& Robazzi, M.L.C.C. (2004). Accidentes de trabajo com material cortopunzante en enfermeras de hospitales. Nure Investigación, 2, 1-7.

Melo, H.R.L.; Brito, C.A.A.; M iranda Filho, D.B.; Souza, S.G.; Henriques, A.P.C. \& Silva, O.B. (2004). Condutas em D oenças infecciosas. Rio de Janeiro, Brasil, Ed. Medsi.

Miola, C.E. (2005). Terapia anti-retroviral: Fatores que interferem na adesão de auxiliares de enfermagem após exposição ocupacional a material biológico. Tese de D outorado, Secretaria de Saúde do Estado de São Paulo, Programa de Pós-graduação em Ciências. São Paulo, Brasil.

Microsoft Corporation (1999). Treinamento rápido em M icrosoft Excel 2000. São Paulo. Ed. Berkeley.

Ministério da Previdência Social. Brasil (2005). Boletim Estatístico da Previdência Social. Disponível em: <http://www.previdencia.gov.br/ 12 02.asp >. Acesso em 20/ago./2005.

Ministério da Saúde. Brasil (1996). Conselho Nacional de Saúde. Diretrizes enormas regulamentadoras depesquisas em seres humanos. Resolução n. 196 de 10/ out./ 1996. In: 0 M undo da Saúde (1997), 21, (1), 52-61.

Ministério da Saúde. Secretaria de Gestão do Trabalho e da Educação na Saúde. Brasil. (2003). Um balanço dos empregos criados na área da saúde (1998-2001). Disponível em: <http://portal.saude.gov.br/portal/saude/ visualizar texto.cfm?idtxt $=13076>$. Acesso em 01/jul/2005.

Monteiro, M.S.; Carnio, A.M .\& Alexandre, N.M.C. (1987). Acidentes de trabalho entre 0 pessoal de enfermagem de um Hospital Universitário. Rev. Bras. Enfermagem, 40(2/3), 8992.

Oliveira, M.G.; Makaron, P.E. \& Morrone, L.C. (1982). Aspectos epidemiológicos: acidentes de trabalho em hospital geral. Rev. Bras. Saúde 0 cup., 10 (40). 737-746.

Parada, E.O.; Alexandre, N.M.C. \& Benatti, M.C.C. (2002). Lesões ocupacionais afetando a coluna vertebral em trabalhadores de enfermagem. Rev. Latino-Am. Enfermagem, 10 (1), 64-9.
Robazzi, M .L.C.C. \& M arziale, M.H .P. (2004). A norma regulamentadora 32 esuas implicações sobre os trabalhadores de enfermagem. Rev. Latino-Am. Enfermagem, 12(5), 834-6.

Robazzi, M .L.C.C. \& BarrosJunior, J.(2005). Proposta brasileira de normatização para os trabalhadores da saúde. Cienc. Enferm, 11 (2), p. 11-5.

Sailer, G.C. (2004). Adesão de trabalhadores de enfermagem ao tratamento com os antiretrovirais pós-exposição ocupacional a material biológico. Dissertação M estrado, Escola de Enfermagem de Ribeirão Preto da Universidade de São Paulo, Ribeirão Preto, Brasil.

Sarquis, L.M.M . (1999). Acidentes de trabalho com instrumentos perfurocortantes: ocorrências entre os trabal hadores de enfermagem. Dissertação de M estrado, Escola de Enfermagem da Universidade de São Paulo, São Paulo, Brasil.

Sêcco, I.A.O. (2002). Acidentes de trabalho com material biológico na equipe de enfermagem de Hospital Escola Público de Londrina - PR, Dissertação M estrado, Centro das Ciências da Saúde, Universidade Estadual de Londrina, Londrina, Brasil.

Sêcco, I.A.O.; Robazzi, M .L.C.; Shimizu, D.S. \& Rúbio, M.M.S. (2005). Epidemiologia do risco de acidentes de trabalho para trabalhadores dehospital público de ensino. In: Congresso Internacional de Saúde da Universidade Estadual de Maringá, I.; Seminário Científico do CCS, V., M aringá, Brasil. Anais. Ed. Universidade Estadual de M aringá.

Silva, A. (1996). Trabalhador de enfermagem na unidade de Centro de $M$ aterial e os acidentes de trabalho. Tese de Doutorado em Enfermagem, Universidade de São Paulo, São Paulo, Brasil.

Silva, V.E.F. (1996). 0 desgaste do trabalhador de enfermagem: estudo da relação trabalho de enfermagem e saúde do trabalhador. Tese de D outorado, Escola de Enfermagem da Universidade de São Paulo, São Paulo, Brasil.

--_---- . (1998). Estudo sobreacidentes de trabalho ocorridos com trabalhadores de enfermagem de um hospital de ensino. Dissertação de M estrado, Escola de Enfermagem da Universidade de São Paulo, São Paulo, Brasil. 
Tomasina, F. \& Gómez Etchebarne, F. (2001). Accidentes laborales en el Hospital de Clínicas. Rev. M éd. Urug., 17 (3), 156-60.

Valenzuela, S.; Sanhueza, O.; Riquelme, N .; N úñez,
M.F.R. (2002). Trabajo hospitalario y salud: La situación de los accidentes del trabajo en el equipo de enfermería de un hospital clínico chileno. Enfermería Clínica 13 (2), 94-102. 